\title{
Uniformly Starlike Functions and Uniformly Convex Functions Associated with the Struve Function
}

\section{Srivastava $\mathrm{HM}^{1 *}$, Murugusundaramoorthy $\mathrm{G}^{2}$ and Janani $\mathrm{T}^{2}$}

${ }^{1}$ Department of Mathematics and Statistics, University of Victoria, Victoria, British Columbia V8W 3R4, Canada

${ }^{2}$ School of Advanced Sciences, VIT University, Vellore 632014, Tamil Nadu, India

\begin{abstract}
In this paper, our goal is to determine sufficient conditions for the family of Struve functions in order to belong to the classes of uniformly convex functions and uniformly starlike functions in the open unit disk U. Several corollaries and consequences of our main results are also derived.
\end{abstract}

Keywords: Starlike functions; Convex functions; Uniformly starlike functions; Uniformly convex functions; Hadamard product (or convolution); Bessel function; Struve function; Generalized hypergeometric function

\section{Introduction and Definitions}

Let $\mathrm{A}$ be the class of functions $\mathrm{f}(\mathrm{z})$ of the form:

$$
f(\mathrm{z})=\mathrm{z}+\sum_{n=2}^{\infty} a_{n} z^{n}
$$

which are analytic in the open unit disk

$$
U=\{z: z \in C \text { and }|z|<1\}
$$

As usual, we denote by $\mathrm{S}$ the subclass of A consisting of functions which are normalized by $\mathrm{f}(0)=0=\mathrm{f}(0)-1$ and are also univalent in $\mathrm{U}$. Moreover, following Silverman [1], we denote by $\mathrm{T}$ the subclass of A consisting of functions of the form:

$$
f(\mathrm{z})=\mathrm{z}-\sum_{n=2}^{\infty} a_{n} z^{n} \quad\left(\mathrm{a}_{\mathrm{n}} \geqq 0\right)
$$

Also, for functions $\mathrm{f} € \mathrm{~A}$ given by (1) and $\mathrm{g} € \mathrm{~A}$ given by

$$
g(\mathrm{z})=\mathrm{z}+\sum_{n=2}^{\infty} b_{n} z^{n}
$$

the Hadamard product (or convolution) of $\mathrm{f}$ and $\mathrm{g}$ is defined by

$$
(\mathrm{f} * \mathrm{~g})(\mathrm{z})=\mathrm{f}(\mathrm{z}) * g(\mathrm{z})=\mathrm{z}+\sum_{n=2}^{\infty} a_{n} b_{n} z^{n}(\mathrm{z} \in \mathrm{U})
$$

A function $\mathrm{f} € \mathrm{~A}$ is said to be starlike of order $\alpha=(0 \leqq \alpha<1)$ in $\mathrm{U}$ if and only if

$$
R\left(\frac{z f^{\prime}(\mathrm{z})}{f(\mathrm{z})}\right)>\alpha \quad(\mathrm{z} \in \mathrm{U} ; 0 \leqq \alpha<1)
$$

This function class is denoted by $S^{*}(\alpha)$ : We also write

$$
S^{*}(0) \equiv S^{*}
$$

where $S^{*}$ denotes the class of functions $f € A$ such that the image $f(U)$ is starlike with respect to the origin. A function $\mathrm{f} € \mathrm{~A}$ is said to be convex of order $\alpha=(0 \leqq \alpha<1)$ in $\mathrm{U}$ if and only if

$$
R\left(1+\frac{z f^{\prime \prime}(\mathrm{z})}{f^{\prime}(\mathrm{z})}\right)>\alpha \quad(\mathrm{z} \in \mathrm{U} ; 0 \leqq \alpha<1)
$$

This function class is denoted by $\mathrm{K}(\alpha)$ : As usual, we write

$$
\mathrm{K}(0) \equiv \mathrm{K}
$$

for the well-known class of convex functions in U. It is a well-established fact that

$$
f(\mathrm{z}) \in \mathrm{K}(\alpha) \Leftrightarrow \mathrm{zf}^{\prime}(\mathrm{z}) \mathrm{S}^{*}(\alpha)
$$

It is well known that the Theory of Special Functions play an important role in Geometric Function Theory, especially in the solution by de Branges [2] of the famous Bieberbachconjecture. There is an extensive literature dealing with geometric properties of different families of special functions, particularly the generalized hypergeometric functions [3-6] and the Bessel functions [7-10]. We recall here the Struve function of order $\mathrm{p}[11,12]$, denoted by $\mathrm{H}_{\mathrm{p}}(\mathrm{z})$ and given by

$$
H_{p}(\mathrm{z})=\sum_{n=0}^{\infty} \frac{(-1)^{\mathrm{n}}}{\Gamma\left(\mathrm{n}+\frac{3}{2}\right) \Gamma\left(p+n+\frac{3}{2}\right)}\left(\frac{z}{2}\right)^{2 n+p+1} \quad(\mathrm{z} \in \mathrm{C}),
$$

which is a particular solution of the following second-order nonhomogeneous differential equation:

$$
z^{2} w^{\prime \prime}(\mathrm{z})+\mathrm{zw}^{\prime}(\mathrm{z})+\left(\mathrm{z}^{2}-\mathrm{p}^{2}\right) w(z)=\frac{4(\mathrm{z} / 2)^{\mathrm{p}+1}}{\sqrt{\pi} \Gamma\left(\mathrm{p}+\frac{1}{2}\right)},
$$

where the parameter $\mathrm{p}$ is an unrestricted real (or complex) number. The solution of the following non-homogeneous differential equation:

$$
z^{2} w^{\prime \prime}(\mathrm{z})+\mathrm{zw}^{\prime}(\mathrm{z})-\left(\mathrm{z}^{2}+\mathrm{p}^{2}\right) w(z)=\frac{4(\mathrm{z} / 2)^{\mathrm{p}+1}}{\sqrt{\pi} \Gamma\left(\mathrm{p}+\frac{1}{2}\right)},
$$

is called the modified Struve function of order $\mathrm{p}$ and is defined by

$$
L_{p}(\mathrm{z})=-\mathrm{ie}^{-\mathrm{i} \mathrm{p} \pi / 2} H_{p}(\mathrm{iz})=\sum_{n=0}^{\infty} \frac{1}{\Gamma\left(\mathrm{n}+\frac{3}{2}\right) \Gamma\left(p+n+\frac{3}{2}\right)}\left(\frac{z}{2}\right)^{2 n+p+1} \quad(\mathrm{z} \in \mathrm{C}),
$$

*Corresponding author: Srivastava HM, Department of Mathematics and Statistics, University of Victoria, Victoria, British Columbia V8W 3R4, Canada, Tel: 1 250-721-7211; E-mail: harimsri@math.uvic.ca

Received July 17, 2014; Accepted August 11, 2014; Published August 19, 2014

Citation: Srivastava HM, Murugusundaramoorthy G, Janani T (2014) Uniformly Starlike Functions and Uniformly Convex Functions Associated with the Struve Function. J Appl Computat Math 3: 180. doi:10.4172/2168-9679.1000180

Copyright: (C) 2014 Srivastava HM, et al. This is an open-access article distributed under the terms of the Creative Commons Attribution License, which permits unrestricted use, distribution, and reproduction in any medium, provided the original author and source are credited. 
Citation: Srivastava HM, Murugusundaramoorthy G, Janani T (2014) Uniformly Starlike Functions and Uniformly Convex Functions Associated with the Struve Function. J Appl Computat Math 3: 180. doi:10.4172/2168-9679.1000180

Page 2 of 4

The following second-order non-homogeneous linear differential equation $[11,12]$

$$
z^{2} w^{\prime \prime}(\mathrm{z})+b z w^{\prime}(\mathrm{z})+\left[\mathrm{cz}^{2}-\mathrm{p}^{2}+(1-\mathrm{b}) \mathrm{p}\right] w(\mathrm{z})=\frac{4(\mathrm{z} / 2)^{\mathrm{p}+1}}{\sqrt{\pi} \Gamma\left(\mathrm{p}+\frac{b}{2}\right)}(\mathrm{b}, \mathrm{p}, \mathrm{c} \in \mathrm{C})
$$

is a natural generalization of the Struve differential equation (4). It is of interest to note that, in its special case when $b=c=1$, we get the Struve equation (4). Also, for $c=-1$ and $b=1$, we get the modified Struve equation (5). We now denote by $\mathrm{w}_{\mathrm{p}, \mathrm{b}, \mathrm{c}}(\mathrm{z})$ the generalized Struve function of order $\mathrm{p}$ given by

$$
w_{p, b, c}(\mathrm{z}):=\sum_{n=0}^{\infty} \frac{(-\mathrm{c})^{\mathrm{n}}}{\left(\mathrm{n}+\frac{3}{2}\right) \Gamma\left(\mathrm{p}+\mathrm{n}+\frac{b+2}{2}\right)}\left(\frac{z}{2}\right)^{2 n+p+1}(\mathrm{z} \in \mathrm{C}),
$$

which is a particular solution of the differential equation (6). Although the series in the above definition is convergent everywhere in $\mathrm{C}$, the function $\mathrm{w}_{\mathrm{p}, \mathrm{c}, \mathrm{c}}$ is generally not univalent in $\mathrm{U}$ : We consider the function $\mathrm{u}_{\mathrm{p}, \mathrm{b}, \mathrm{c}}$ defined by

$$
u_{p, b, c}(\mathrm{z})=2^{p} \sqrt{\pi} \Gamma\left(\mathrm{p}+\frac{b+2}{2}\right) z^{-\frac{p+1}{2}} w_{p, b, c}(\sqrt{z})(\sqrt{1}:=1),
$$

by using the well-known Pochhammer symbol (or the shifted factorial) ()n given, in terms of the familiar Gamma function, by

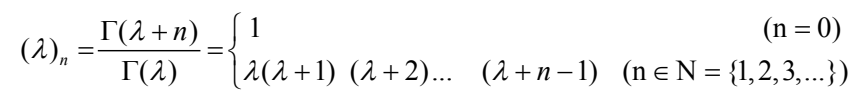

We can express $\mathrm{u}_{\mathrm{p}, \mathrm{b}, \mathrm{c}}(\mathrm{z})$ as follows:

$$
u_{p, b, c}(\mathrm{z})=\sum_{n=0}^{\infty} \frac{\left(-\frac{c}{4}\right)^{\mathrm{n}}}{(m)_{n}\left(\frac{3}{2}\right)_{n}} z^{n}=b_{0}+b_{1} z+b_{2} z^{2}+\ldots+b_{n} z^{n}+\ldots,
$$

Where

$$
m:=p+\frac{b+2}{2} \neq 0,-1,-2, \ldots .
$$

This function $\mathrm{u}_{\mathrm{p}, \mathrm{b}, \mathrm{c}}(\mathrm{z})$ is analytic on $\mathrm{C}$ and satisfies the following second-order in homogeneous linear differential equation:

$$
4 z^{2} u^{\prime \prime}(z)+2(2 p+b+3) z^{\prime}(z)+(c z+2 p+b) u(z)=2 p+b
$$

For our convenience throughout this paper, we use the following notations:

$$
w_{p, b, c}(\mathrm{z})=: \mathrm{w}_{\mathrm{p}}(\mathrm{z}), \mathrm{u}_{\mathrm{p}, \mathrm{b}, \mathrm{c}}(\mathrm{z})=: \mathrm{u}_{\mathrm{p}}(\mathrm{z}) \text { and } \mathrm{m}=\mathrm{p}+\frac{b+2}{2}
$$

Moreover, if $\mathrm{c}<0$ and $\mathrm{m}>0$, we let

$$
\begin{aligned}
& z u_{p}(\mathrm{z})=\mathrm{z}+\sum_{n=2}^{\infty} \frac{\left(\frac{-c}{4}\right)^{n-1}}{(\mathrm{~m})_{\mathrm{n}-1}\left(\frac{3}{2}\right)_{n-1}} z^{n} \\
& =\mathrm{z}+\sum_{n=2}^{\infty} b_{n}-1 z^{n}
\end{aligned}
$$

And
$\Psi(\mathrm{z})=\mathrm{z}\left[2-\mathrm{u}_{\mathrm{p}}(\mathrm{z})\right]=\mathrm{z}-\sum_{n=2}^{\infty} \frac{\left(\frac{-c}{4}\right)^{n-1}}{(\mathrm{~m})_{\mathrm{n}-1}\left(\frac{3}{2}\right)_{n-1}} \mathrm{z}^{n}$
Recently, Yagmur and Orhan $[11,12]$ determined various sufficient conditions for the parameters $\mathrm{p} ; \mathrm{b}$ and $\mathrm{c}$ such that the functions $\mathrm{u}_{\mathrm{p}}(\mathrm{z})$ or $\mathrm{z} \rightarrow \mathrm{zu}_{\mathrm{p}}(\mathrm{z})$ are univalent, starlike, convex and close-to-convex in the open unit disk U. Here, in our present investigation, we consider the following subclasses which were studied earlier by Rosy et al. [13] and Subramanian et al. [14].

Definition 1: [13] For $\beta>0$; a function $\mathrm{f} € \mathrm{~A}$ of the form (1) is said to be in the subclass $\operatorname{USD}(\beta)$ of the normalized univalent function class of $S$ if it satisfies the following inequality:

$$
R\left(\mathrm{f}^{\prime}(\mathrm{z})\right) \geqq \beta\left|z f^{\prime \prime}(\mathrm{z})\right| \quad(\mathrm{z} \in \mathrm{U}) \mid
$$

Definition 2: [14] For $\beta>0$, a function $\mathrm{f} € \mathrm{~T}$ of the form (2) is said to be in the subclass UST N $(\beta)$ if it satisfies the following analytic criterion:

$$
R\left(\frac{f(\mathrm{z})-\mathrm{f}(\xi)}{(\mathrm{z}-\xi) \mathrm{f}^{\prime}(\mathrm{z})}\right)>\beta \quad((\mathrm{z}, \xi) \in \mathrm{U} \times \mathrm{U})
$$

Motivated by several earlier results on connections between various subclasses of analytic and univalent functions by using hypergeometric functions $[3,4,6,15]$ and by the recent investigations of Baricz [7-9], in the present paper we determine sufficient conditions for the function $\mathrm{h}_{\mu}(\mathrm{z})$ given by

$$
\begin{aligned}
& h_{\mu}(\mathrm{z}):=(1-\mu) \mathrm{zu}_{\mathrm{p}}(\mathrm{z})+u \mathrm{zu}_{\mathrm{p}}^{\prime}(\mathrm{z}) \\
& =z+\sum_{n=2}^{\infty}(1+\mathrm{n} \mu-\mu) \frac{\left(\frac{-c}{4}\right)^{n-1}}{(\mathrm{~m})_{\mathrm{n}-1}\left(\frac{3}{2}\right)_{n-1}} z^{n} \quad(0 \leqq \mu \leqq 1)
\end{aligned}
$$

in order to belong to the above-defined classes $\operatorname{USD}(\beta)$ and UST N $(\beta)$ :

\section{A Pair of Useful Lemmas}

To prove the main results in our present investigation, we shall need each of the following lemmas.

Lemma 1: [13] A function $f$ of the form (1) is in the class $\operatorname{USD}(\beta)$ if

$$
\sum_{n=2}^{\infty}\left[\mathrm{n}(1-\beta)+n^{2} \beta\right] \cdot\left|a_{n}\right| \leqq 1
$$

Lemma 2: [14] A function $f$ of the form (2) is in the class UST N( $\beta)$ if

$$
\sum_{n=2}^{\infty}[\mathrm{n}(3-\beta)-2] \cdot\left|a_{n}\right| \leqq 1-\beta
$$

\section{Main Results and their Consequences}

Our first main result is asserted by Theorem 1 below.

Theorem 1: Let $c<0$ and $m>0$. Then $h_{\mu}(z) 2 \operatorname{USD}(\mu)$ if

$\mu \beta u_{p}^{\prime \prime \prime}(1)+(\mu+\beta+2 \mu \beta) u_{p}^{\prime \prime}(1)+[2(\mu+\beta-\mu \beta)+1] \mathrm{u}_{p}^{\prime}(1)+\mathrm{u}_{\mathrm{p}}(1) \leqq 2$

Proof: Let

$z u_{p}(\mathrm{z})=\mathrm{z}+\sum_{n=2}^{\infty} \frac{\left(-\frac{c}{4}\right)^{n-1}}{(\mathrm{~m})_{\mathrm{n}-1}\left(\frac{3}{2}\right)_{n-1}} z^{n}$

Upon taking $\mathrm{z}=1$, we have 


$$
u_{p}(1)-\mid 1=\sum_{n=2}^{\infty} \frac{\left(-\frac{c}{4}\right)^{n-1}}{(\mathrm{~m})_{\mathrm{n}-1}\left(\frac{3}{2}\right)_{n-1}}
$$

Differentiating $\mathrm{zu}_{\mathrm{p}}(\mathrm{z})$ with respect to $\mathrm{z}$ and setting $\mathrm{z}=1$, we have

$$
z u_{p}^{\prime}(z)+u_{p}(\mathrm{z})=1+\sum_{n=2}^{\infty} n \frac{\left(-\frac{c}{4}\right)^{n-1}}{(\mathrm{~m})_{\mathrm{n}-1}\left(\frac{3}{2}\right)_{n-1}} z^{n-1},
$$

so that

$$
u_{p}^{\prime}(1)+u_{p}(1)-1+\sum_{n=2}^{\infty} n \frac{\left(-\frac{c}{4}\right)^{n-1}}{(\mathrm{~m})_{\mathrm{n}-1}\left(\frac{3}{2}\right)_{n-1}}
$$

Further, by differentiating $\mathrm{zu}_{\mathrm{p}}(\mathrm{z})+\mathrm{u}_{\mathrm{p}}(\mathrm{z})$ with respect to $\mathrm{z}$ and taking $\mathrm{z}=1$, we get

$$
z u_{p}^{\prime \prime}(z)+2 u_{p}^{\prime}(z)=\sum_{n=2}^{\infty} n(\mathrm{n}-1) \frac{\left(-\frac{c}{4}\right)^{n-1}}{(\mathrm{~m})_{\mathrm{n}-1}\left(\frac{3}{2}\right)_{n-1}} z^{n-2}
$$

So that

$$
u_{p}^{\prime \prime}(1)+2 u_{p}^{\prime}(1)=\sum_{n=2}^{\infty} n(\mathrm{n}-1) \frac{\left(-\frac{c}{4}\right)^{n-1}}{(\mathrm{~m})_{\mathrm{n}-1}\left(\frac{3}{2}\right)_{n-1}}
$$

A further differentiation similarly yields

$$
z u_{p}^{\prime \prime \prime}(z)+3 u_{p}^{\prime \prime \prime}(1)=\sum_{n=2}^{\infty} n(\mathrm{n}-1)(\mathrm{n}-2) \frac{\left(-\frac{c}{4}\right)^{n-1}}{(\mathrm{~m})_{\mathrm{n}-1}\left(\frac{3}{2}\right)_{n-1}} z^{n-3}
$$

And

$$
u_{p}^{\prime \prime \prime}(1)+3 u_{p}^{\prime \prime \prime}(1)=\sum_{n=2}^{\infty} n(\mathrm{n}-1)(\mathrm{n}-2) \frac{\left(-\frac{c}{4}\right)^{n-1}}{(\mathrm{~m})_{\mathrm{n}-1}\left(\frac{3}{2}\right)_{n-1}}
$$

Since $h_{\mu}(z) € U S D(\beta)$; by virtue of Lemma 1 it suffices to show that $\sum_{n=2}^{\infty}(1+\mathrm{n} \mu-\mu)\left(\mathrm{n}\left(1-\beta+n^{2} \beta\right] \frac{\left(-\frac{c}{4}\right)^{n-1}}{(\mathrm{~m})_{\mathrm{n}-1}\left(\frac{3}{2}\right)_{n-1}} \leqq 1\right.$

We now let $S(\lambda, \beta, \alpha)=\sum_{n=2}^{\infty}(1+\mathrm{n} \mu-\mu)\left(\mathrm{n}\left(1-\beta+n^{2} \beta\right] \frac{\left(-\frac{c}{4}\right)^{n-1}}{(\mathrm{~m})_{\mathrm{n}-1}\left(\frac{3}{2}\right)_{n-1}}\right.$ so that

$$
S(\lambda, \beta, \alpha)=\mu \beta \sum_{n=2}^{\infty} n^{3} \frac{\left(-\frac{c}{4}\right)^{n-1}}{(\mathrm{~m})_{\mathrm{n}-1}\left(\frac{3}{2}\right)_{n-1}} \quad+(\mu+\beta-\mu \beta) \sum_{n=2}^{\infty} n^{2} \frac{\left(-\frac{c}{4}\right)^{n-1}}{(\mathrm{~m})_{\mathrm{n}-1}\left(\frac{3}{2}\right)_{n-1}}
$$

$+(1+\mu-\beta+\mu \beta) \sum_{n=2}^{\infty} n \frac{\left(-\frac{c}{4}\right)^{n-1}}{(\mathrm{~m})_{\mathrm{n}-1}\left(\frac{3}{2}\right)_{n-1}}$

Writing

$n^{3}=n(\mathrm{n}-1)(\mathrm{n}-2)+3 \mathrm{n}(\mathrm{n}-1)+\mathrm{n}$ and $\mathrm{n}^{2}=n(\mathrm{n}-1)+\mathrm{n} \quad$ we get

$S(\mathrm{n}, \lambda, \beta, \alpha)=\mu \beta \sum_{n=2}^{\infty} n(\mathrm{n}-1)(\mathrm{n}-2) \frac{\left(-\frac{c}{4}\right)^{n-1}}{(\mathrm{~m})_{\mathrm{n}-1}\left(\frac{3}{2}\right)_{n-1}}$

$+(\mu+\beta-\mu \beta) \sum_{n=2}^{\infty} n(\mathrm{n}-1) \frac{\left(-\frac{c}{4}\right)^{n-1}}{(\mathrm{~m})_{\mathrm{n}-1}\left(\frac{3}{2}\right)_{n-1}}$

$+\sum_{n=2}^{\infty} n \frac{\left(-\frac{c}{4}\right)^{n-1}}{(\mathrm{~m})_{\mathrm{n}-1}\left(\frac{3}{2}\right)_{n-1}}$

From (15), (17) and (19), we get

$\left.S(\mathrm{n}, \lambda, \beta, \alpha)=\mu \beta\left[\mathrm{u}_{p}^{\prime \prime \prime}(1)+3_{p}^{\prime \prime \prime}(1)\right]+(\mu+\beta-\mu \beta)\left[\mathrm{u}_{p}^{\prime \prime}(1)+2 u_{\mathrm{p}}^{\prime}(1)\right]+u_{\mathrm{p}}^{\prime}(1)-1\right]$ $\mu \beta \mathrm{u}_{p}^{\prime \prime \prime}(1)+(\mu+\beta+2 \mu \beta) \mathrm{u}_{p}^{\prime \prime}(1)+[2(\mu+\beta-\mu \beta+1)] \mathrm{u}_{p}^{\prime}(1)+\mathrm{u}_{\mathrm{p}}(1)-1$

But this last expression is bounded above by 1 if (12) holds true. Thus the proof of Theorem 1 is completed.

Theorem 2: Let $c<0$ and $m>0$. Then $\mathrm{zu}_{\mathrm{p}}(\mathrm{z}) € \operatorname{USD}(\beta)$ if

$$
\beta \mathrm{u}_{p}^{\prime \prime}(1)+u_{p}^{\prime}(1)+\mathrm{u}_{\mathrm{p}}(1) \leqq 2
$$

Proof: By virtue of Lemma 1, it suffices to show that

$$
\sum_{n=2}^{\infty}\left[n(1-\beta)+\left(\mathrm{n}^{2} \beta\right) \frac{\left(-\frac{c}{4}\right)^{n-1}}{(\mathrm{~m})_{\mathrm{n}-1}\left(\frac{3}{2}\right)_{n-1}} \leqq 1\right.
$$

We note that

$h_{0}(\mathrm{z})=\mathrm{zu}_{\mathrm{p}}(\mathrm{z})$

Hence, by taking $\mu=0$ in (20), we get the above inequality. Therefore, by setting $\beta=0$ in Theorem 1, we get the desired result given in (21).

Corollary 1: Let $c<0$ and $m>0$. Then $\mathrm{zu}_{\mathrm{p}}(\mathrm{z}) € \operatorname{USD}(0)$ if and only if

$u_{p}^{\prime}(1)+u_{p}(1) \leqq 2$

Theorem 3: Let $c<0$ and $m>0$. Then $h_{\mu}(z) € \operatorname{UST} N(\beta)$ if

$\mu(3-\beta) \mathrm{u}_{p}^{\prime \prime}(1)+[(3-\beta)(2 \mu+1)-2 \mu] \mathrm{u}_{p}^{\prime}(1)+(1-\beta) \mathrm{u}_{\mathrm{p}}(1) \leqq 2(1-\beta)$

Proof: By virtue of Lemma 2, it suffices to show that 
Citation: Srivastava HM, Murugusundaramoorthy G, Janani T (2014) Uniformly Starlike Functions and Uniformly Convex Functions Associated with the Struve Function. J Appl Computat Math 3: 180. doi:10.4172/2168-9679.1000180

$\sum_{n=2}^{\infty}(1+n \mu-\mu)[(3-\beta) \mathrm{n}-2] \frac{\left(-\frac{c}{4}\right)^{n-1}}{(\mathrm{~m})_{\mathrm{n}-1}\left(\frac{3}{2}\right)_{n-1}} \leqq 1-\alpha$

We now let

$S(\mathrm{n}, \lambda, \beta, \alpha)=\sum_{n=2}^{\infty}(1+\mathrm{n} \mu-\mu)[(3-\beta) n-2] \frac{\left(-\frac{c}{4}\right)^{n-1}}{(\mathrm{~m})_{\mathrm{n}-1}\left(\frac{3}{2}\right)_{n-1}}$,

That is

$$
\begin{aligned}
& S(\mathrm{n}, \lambda, \beta, \alpha)=\sum_{n=2}^{\infty} \mu(3-\beta) \frac{\left(-\frac{c}{4}\right)^{n-1}}{(\mathrm{~m})_{\mathrm{n}-1}\left(\frac{3}{2}\right)_{n-1}}, \\
& +[(3-\beta)(1-\mu)-2 \mu] \sum_{n=2}^{\infty} n \frac{\left(-\frac{c}{4}\right)^{n-1}}{(\mathrm{~m})_{\mathrm{n}-1}\left(\frac{3}{2}\right)_{n-1}}, \\
& -2(1-\mu) \sum_{n=2}^{\infty} \frac{\left(-\frac{c}{4}\right)^{n-1}}{(\mathrm{~m})_{\mathrm{n}-1}\left(\frac{3}{2}\right)_{n-1}},
\end{aligned}
$$

which, upon writing $\mathrm{n}^{2}=\mathrm{n}(\mathrm{n}-1)+\mathrm{n}$, yields

$$
\begin{aligned}
& S(\mathrm{n}, \lambda, \beta, \alpha)=\mu(3-\beta) \sum_{n=2}^{\infty} n(\mathrm{n}-1) \frac{\left(-\frac{c}{4}\right)^{n-1}}{(\mathrm{~m})_{\mathrm{n}-1}\left(\frac{3}{2}\right)_{n-1}}, \\
& +(3-\beta-2 \mu) \sum_{n=2}^{\infty} n \frac{\left(-\frac{c}{4}\right)^{n-1}}{(\mathrm{~m})_{\mathrm{n}-1}\left(\frac{3}{2}\right)_{n-1}}-2(1-\mu) \sum_{n=2}^{\infty} \frac{\left(-\frac{c}{4}\right)^{n-1}}{(\mathrm{~m})_{\mathrm{n}-1}\left(\frac{3}{2}\right)_{n-1}}
\end{aligned}
$$

From (13), (15) and (17), we get

$$
\begin{aligned}
& S(\mathrm{n}, \lambda, \beta, \alpha)=\mu(3-\beta)\left[\mathrm{u}_{p}^{\prime \prime}(1)+2 \mathrm{u}_{p}^{\prime}(1)\right]+(3-\beta-2 \mu)\left[\mathrm{u}_{p}^{\prime}(1)+\mathrm{u}_{\mathrm{p}}(1)-1\right], \\
& -2(1-\mu)\left[\mathrm{u}_{\mathrm{p}}(1)-1\right] \\
& =\mu(3-\beta) \mathrm{u}_{p}^{\prime \prime}(1)+[(3-\beta)(2 \mu+1)-2 \mu] \mathrm{u}_{p}^{\prime}(1)+(1-\beta)\left[\mathrm{u}_{\mathrm{p}}(1)-1\right]
\end{aligned}
$$

But this last expression is bounded above by 1- $\beta$ if the condition (23) holds true. Thus the proof of Theorem 3 is completed.

By taking $\mu=0$ in Theorem 3, we can easily deduce the following corollary which we state here without proof.

Corollary 2: Let $c<0$ and $\mathrm{m}>0$. Then $\mathrm{zu}_{\mathrm{p}}(\mathrm{z}) 2$ UST N $(\beta)$ if

$(3-\beta) \mathrm{u}_{p}^{\prime}(1)+(1-\beta) \mathrm{u}_{\mathrm{p}}(1) \leqq 2(1-\beta)$

Furthermore; $\mathrm{zu}_{\mathrm{p}}(\mathrm{z}) 2$ UST N(0) if

$$
3 u_{p}^{\prime}(1)+u_{p}(1) \leqq 2
$$

\section{References}

1. Silverman $H$ (1975) Univalent functions with negative coefficients. Proc Amer Math Soc 51: 109-116.

2. de Branges $L$ (1985) A proof of the Bierberbach conjucture. Acta Math 154 137-152.

3. Cho NE, Woo SY, Owa S (2002) Uniform convexity properties for hypergeometric functions. Fract Calc Appl Anal 5: 303-313.

4. Merkes EP, Scott WP (1961) Starlike hypergeometric functions. Proc Amer Math Soc 12: 885-888.

5. Owa S, Srivastava HM (1987) Univalent and starlike generalized hypergeometric functions. Canad J Math 39 1057-1077.

6. Silverman H (1993) Starlike and convexity properties for hypergeometric functions. J Math Anal Appl 172: 574-581.

7. Baricz A (2010) Geometric properties of generalized Bessel functions. Publ Math Debrecen 73: 155-178.

8. Baricz A (2006) Geometric properties of generalized Bessel functions of complex order. Mathematica (Cluj) 48: 13-18.

9. Baricz A (2010) Generalized Bessel Functions of the First Kind. Lecture Notes in Mathematics, Springer-Verlag, Berlin, Heidelberg and New York, 2010.

10. Mondal SR, Swaminathan A (2012) Geometric properties of generalized Besse functions. Bull Malays Math Sci Soc 35: 179-194.

11. Orhan H, Yagmur N (2014) Geometric properties of generalized Struve functions. Ann Alexandru Ioan Univ Math 60.

12. Yagmur N, Orhan $H$ (2013) Starlikeness and convexity of generalized Struve functions. Abstr Appl Anal Article ID 954513, 1-6.

13. Rosy T, Stephen BA, Subramanian KG, Silverman H (2000) Classes of convex functions. Internat J Math Math Sci 23: 819-825.

14. Subramanian KG, Murugusundaramoorthy $G$, Balasubrahmanyam $P$, Silverman $\mathrm{H}$ (1995) Subclasses of uniformly convex and uniformly starlike functions. Math Japon 42: 517-522.

15. Srivastava HM, Murugusundaramoorthy G, Sivasubramanian S (2007) Hypergeometric functions in the parabolic starlike and uniformly convex domains. Integral Transforms Spec Funct 18: 511-520. 